\title{
Study on the Causes and Countermeasures of Medical Complaints During the Outbreak of Novel Coronavirus Disease
}

\author{
Wenhong Guo ${ }^{1}$, Lu Zhang ${ }^{2}$, Zhangqing Cui ${ }^{2}$, Lingjie Kong ${ }^{2}$, Songyue $\mathrm{He}^{2^{* *}}$ \\ ${ }^{1}$ Medical Doctor-Patient Coordination Office, Tiantan Hospital of Capital Medical University, Beijing, 100160 \\ ${ }^{2}$ Beijing Fayuan Forensic Science Evidence Expertise Center; Beijing, 100062 \\ Email: Songyue-He@163.com
}

\begin{abstract}
The aim is to investigate the medical complaints status and coping styles during the outbreak of the novel coronavirus diseases. Methods: 86 cases medical complaints of uninfected novel coronavirus disease, which were collected from one hospital at Beijing city during the January-March 2020, have been retrospectively analyzed. The number, types and causes, department distribution have been evaluated at different stages. Results: During the epidemic, medical complaints are still one of the severe problems faced by hospitals; the number of medical complaints was significantly higher than that during the non-epidemic; Emergency, Neurology and Neurosurgery were the top three departments. Failure to admission to hospital as scheduled or as expected was an important and special reason in the medical complaints. Most of medical complaints were gain understanding and cooperation by effective communication, and only 4 cases of complaints were application for Legal mediation procedure legal. Conclusion: The medical complaint during the outbreak is a serious problem facing by hospitals, which should be given the necessary attention and positive response to reduce the risk of related medical litigation cases at post-epidemic stage.

Keywords: novel coronavirus disease, hospital, medical complaints, precautionary measures
\end{abstract}

\section{Introduction}

The novel coronavirus pneumonia (Corona Virus Disease 2019, COVID-19), which has appeared in Wuhan, China since 12th, 2019, has been pandemic worldwide and caused profound social and medical problems. It is listed by the World Health Organization as "Public health emergency of International Concerned" ${ }^{[1]}$, medical diagnosis and treatment activities and hospital management is confronted with a series of new problems and challenges. In this regard, the article has put forward the investigation of the medical complaint cases of patients with non-novel coronavirus pneumonia, understanding the causes and characteristics of medical complaints during the epidemic, and to provide reference for the prevention and handling of medical legal issues involved in public health emergencies.

\section{Data and methods}

A retrospective analysis method was used to study about 86 cases of medical complaints received by doctor-patient coordination office in a tertiary first-class hospital in Beijing from January to mid-March 2020. With reference to the clinic guidelines for novel coronavirus pneumonia (First edition), January 15, 2020 was used as the time-point to distinguish the epidemic and non-epidemic periods in Beijing ${ }^{[2]}$. Through induction and analysis of the number of complaints, growth trends, distribution of departments, cases types, specific reasons and results of cases, the characteristics of medical complaints and effectiveness of measures in different periods were further evaluated.

\section{Results}

\subsection{Cases of medical complaints in difference stages}

A total of 86 medical complaint cases were received from January to mid-March 2020, mainly involves to the diagnosis and treatment activities, medical service attitudes, and medical informed consent. Among these cases, there were 14 complaint cases during the non-epidemic period before mid-January, and a total of 74 complaint cases during the epidemic period from mid-January to mid-March. The specific statistical result as follows: 14 cases from the beginning of January to the middle of January; 8 cases from the middle of January to the end of January, 15 cases before the middle of February, 27 cases after the middle of February, and 22 cases before the middle of March. 


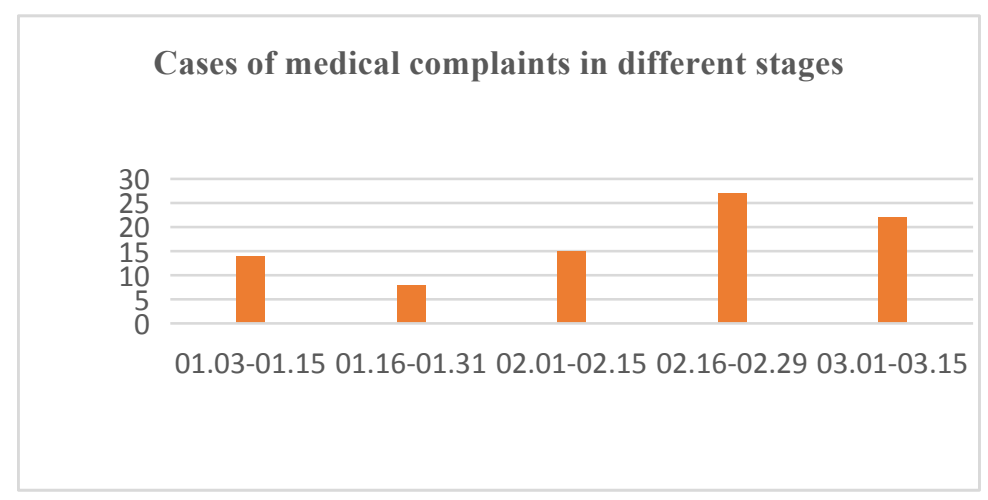

Figure 1. Trend growth in cases of medical complaints

\subsection{Reasons for medical complaints and distribution of departments}

The complaints department mainly includes 32 emergency departments ( 9 cases in the pre-epidemic period), 12 cases in the neurology department ( 2 cases in the pre-epidemic period), 12 cases in neurosurgery ( 1 case in the pre-epidemic period), 9 comprehensive outpatient department and 5 cases in pediatric. During the epidemic, the number of complaints associated with failure to get in hospital as scheduled or as expected were increased significantly. However, there had no obvious change in complaints about medical treatment behavior, doctor-patient communication disputes and medical service between non-epidemic and epidemic period ( Table 1)

Table 1. Reasons for medical complaints during the epidemic

\begin{tabular}{|c|c|c|}
\hline Types of medical complaints & Major complaints & $\begin{array}{c}\text { Number of } \\
\text { cases }\end{array}$ \\
\hline \multirow[t]{3}{*}{$\begin{array}{l}\text { Disputes on doctor-patient } \\
\text { communication effect }\end{array}$} & No inquiries received about scheduled clinical examination many times & 1 \\
\hline & Unpatient answers question to patient in emergency & 1 \\
\hline & Simple explanation transfer from ICU to general wards & 1 \\
\hline \multirow[t]{2}{*}{$\begin{array}{l}\text { Disputes on } \\
\text { admitted patients }\end{array}$} & Failure to be admit patients due to epidemic situation & 45 \\
\hline & Failure to be admit critical patient in time & 3 \\
\hline \multirow[t]{2}{*}{$\begin{array}{l}\text { Question the quality of medical } \\
\text { services }\end{array}$} & Unsatisfactory medical attitude in clinical ultrasound examination & 1 \\
\hline & Worried about nosocomial infection during the epidemic & 4 \\
\hline \multirow[t]{5}{*}{$\begin{array}{l}\text { Disputes on clinical diagnosis and } \\
\text { treatment }\end{array}$} & Over-treatment to patient with nephrolithiasis & 1 \\
\hline & Post-operative incision infection in patient with tumor & 1 \\
\hline & Medical malpractice in patient with cerebrovascular diseases & 1 \\
\hline & Insufficient blood transfusion after cardiac operative & 1 \\
\hline & Unreasonable CT multiple checks during the epidemic & 1 \\
\hline
\end{tabular}


Table 2. Main reasons associated with failure to be admitted during the epidemic

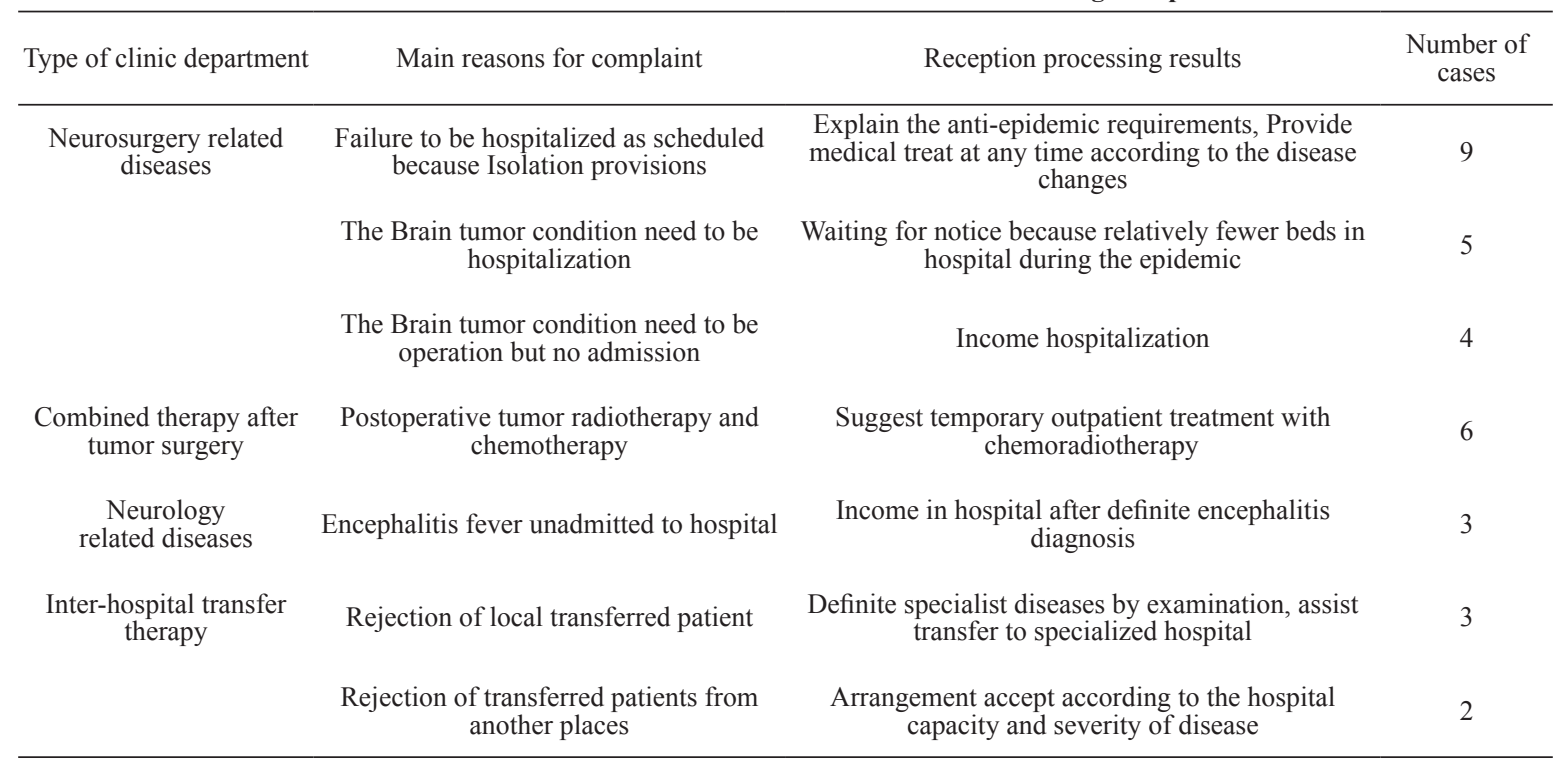

The common reasons associated with failure to be admitted in time during the epidemic were showed in Table 2 . Isolation requirements of epidemic prevention and control, postoperative expected hospitalization for radiotherapy and chemotherapy, delay surgery in scheduled were observed. In this regard, by evaluating surgery time limit, priority for emergency surgery patients, reasonable arrangement selective and limit operation in accordance with isolation time requirements, and outpatient treatment as mainly alternative measures for post-operative chemoradiotherapy patients, most complaints have been resolved with cooperation. Only 4 cases applied for legal mediation procedures.

\section{Discussions}

Receiving and handling medical complaints has always been a complex task faced by the hospitals, and it is also an important part to reduce medical litigation and resolve the contradiction between doctors and patients, ensure clinical diagnosis and treatment activities and the hospital's work daily in a stable, orderly and effective state. The new characteristics of medical complaints during the novel coronavirus pneumonia should be attention and reflection.

In this study, Table 1 shows the common causes of medical complaints during pre-epidemic, which is basically the same as those of other scholars ${ }^{[3,4]}$, mainly focusing on disputes between doctor-patient communication effects, medical service quality challenge, clinical diagnosis and treatment behavior disputes, outpatient work management systems, and hospital prescription management systems, suggesting that medical worker still need to pay attention to these complaint causes, develop the legal and medical skills in doctor-patient communication, improve the medical services quality.

The epidemic of novel coronavirus pneumonia appeared in Wuhan, China, has experienced three stages: local outbreak, community outbreak and widespread transmission ${ }^{[5]}$. On January 15, 2020, the National Health and Welfare Commission of China issued the first edition of the "New Coronavirus Pneumonia Diagnosis and Treatment Program (Trial)" ${ }^{[6]}$, the prevention and control of national epidemics has gradually been standardized and systematized. However, due to provide some medical staff to support the fight against the epidemic in Hubei Province, increasing the beds of Infectious disease in hospitals, management and control of hospital acquired infections, prevention and control measures for personnel flow, some patients can't be admitted to hospitals as scheduled to become a serious status. The results of this study reflect a significant increase trend in the number of epidemic-related medical complaints in hospitals from midJanuary 2020, including specific medical complaints related to the admission of patients to hospital.

During the period of epidemic prevention and control, non-new coronavirus pneumonia patients failed to stay in hospital as scheduled or as expected, it will be difficult to avoid the increase of the mortality rate or aggravation of the disease in these patients, and will be increase the number of litigation cases of doctor-patient disputes in future. Therefore, the hospital has taken a variety of measures to resolve the contradiction. For example, set up a dedicated post to receive complaints, explain the relevant policies and measures of government and CDC in Medical Doctor-Patient Coordination Office; actively evaluate the patient's condition and the operation time limit, and receive hospitalization in batches; 
additional screening clinics, transition observation and isolation wards, strengthen the investigation of epidemiological history, nucleic acid test of new coronavirus pneumonia and CT examination of patients before hospitalization; control the infection in the hospital, decline the patient's family visit; coordination the transfer treatment for patients with other specialist diseases (e.g. pregnant women, kidney dialysis, hepatitis B patients, etc.); enhance the ability of outpatient clinic to receive radiotherapy, chemotherapy and rehabilitation for postoperative patients to reduce the density of inpatients; keep close contact with medical experts and colleagues engaged in judicial identification of medical damage, listen to relevant suggestions on prevention of medical disputes, improve legal requirements on procedural content of medical notification and clinical diagnosis and treatment, and make good processes for collection and preservation of corresponding medical evidence. It alleviated the patients' anxiety and worry about the diagnosis and treatment of the disease. Most of the complaint cases were understood and cooperated. Only four patients proposed to be transfer to the Medical Dispute Mediation Center or the court of the jurisdiction for legal treatment.

It should be noted that recently, some local high-level people's courts such as Zhejiang, Jiangsu and Heilongjiang province have issued legal documents for the trial of relevant civil and commercial cases during the new coronary pneumonia epidemic, and the acceptance and trial of relevant medical disputes involved during the period of epidemic have been explained. It can be predicted that related medical disputes in the later stage of the epidemic may become a hot spot in civil litigation cases. Therefore, in addition to the need to actively prevent and control and treat patients with new coronavirus pneumonia, the necessary measures should also be taken to ensure the orderly diagnosis and treatment the related disease in non-new coronavirus pneumonia patients during the period of epidemic.

The clinical studies at present have shown ${ }^{[7,8]}$ that major public health events caused by coronavirus have a great possibility of recurrence or coming in a new form, and human society will still face serious social, medical and legal challenges arising from the coronavirus epidemic in the future. Although we reported that the hospital has taken some measures to reduce the adverse effects of new coronavirus pneumonia during the outbreak, but we also believe that there are still unsatisfactory aspects of the response to the outbreak by relying solely on the hospitals themselves. It is also necessary to establish an emergency medical system with rapid, professional, joint and cooperative regional medical institutions, so as to transmit new information on the diagnosis and treatment of the epidemic in a timely manner on the information platform set up by the local health command center, and coordinate the medical management-coordinationcooperation model of medical treatment such as transfer and consultation among medical institutions.

\section{References}

[1] World Health Organization. International health regulations emergency committee on novel coronavirus in China. 2020. Available from: https://www.who.int/news-room/events/detail.

[2] Shen Yinzhong, Lu Hongzhou. Raising awareness of the diagnosis and treatment of new coronavirus infections. Chinese Journal of Infectious Diseases. 2020; 38. Available from: http: //rs.yiigle.com/yufabiao/1179579.htm.

[3] Tian Xin, Yu Yabin, Liang Li, Xin Youqing. A comparative study of medical complaints and disputes between to tertiary general hospitals in Beijing and Lhasa. Chin J Hosp Admin. 2017; 33(09): 704-701.

[4] Dai Juan. Cause analysis and countermeasure research of medical disputes and complaints in new normal. Jiangsu Health System Management. 2019; 30(1): 35-38.

[5] Special Expert Group for Control of the Epidemic of Novel Coronavirus Pneumonia of the Chinese Preventive Medicine. An update on the epidemiological characteristics of novel coronavirus pneumonia. Chin J Epidemiol. 2020; 41(02): 139-144. Available from: doi: 10.3760/cma.j.issn.2020.02.002.

[6] Jiang Rongmeng. Process of guidelines for diagnosis and treatment of new coronavirus pneumonia trial version 1-6. Chinese Journal of Infectious Diseases. 2020; 38. Available from: http://rs.yiigle.com/yufabiao/1182640.htm.

[7] Zhang Wenhong. New Coronavirus Rediscovery and the Future of Prevention and Control of New Infectious Diseases. Chinese Communicable DiseasesChi. 2020; 38(01): 3-5. Available from: doi: 10.3760/ cma.j.issn.2020.01.002.

[8] Epidemiology Working Group for NCIP Epidemic Response, Chinese Center for Disease Control and Prevention. The epidemiological characteristics of an outbreak of 2019 novel coronavirus diseases in China. Chin J Epidemiol. 2020; 41(02): 145-151. Available from: doi: 10.3760/cma.j.issn.2020.02.003. 